University of Wollongong

Research Online

Faculty of Engineering and Information

Faculty of Engineering and Information

Sciences - Papers: Part B

Sciences

2012

\title{
Whole-body torques for generating complex movements in humans and humanoids
}

Layale Saab

CNRS Centre National de la Recherche Scientifique

Nicolas Mansard

CNRS Centre National de la Recherche Scientifique

Philippe Souères

LAAS-CNRS Toulouse France

J-Y Fourquet

Universite de Toulouse

Manish Sreenivasa

University of Wollongong, manishs@uow.edu.au

See next page for additional authors

Follow this and additional works at: https://ro.uow.edu.au/eispapers1

Part of the Engineering Commons, and the Science and Technology Studies Commons

Research Online is the open access institutional repository for the University of Wollongong. For further information contact the UOW Library: research-pubs@uow.edu.au 


\title{
Whole-body torques for generating complex movements in humans and humanoids
}

\author{
Abstract \\ This paper considers the problem of identifying the control torques associated with the generation of \\ complex movements in an anthropomorphic system. We present a generic motion generation scheme for \\ humanoid robots. Then we use the torques estimated from human motion capture and force sensor \\ measurements, to compare with similar movements simulated on a humanoid robot. The general features \\ of movement during a sequence of reaching tasks are analyzed. In particular we compare kinematics- \\ based and dynamics-based movements. Finally, the variation of torques at the different joints are \\ compared and discussed.

\section{Keywords} \\ movements, whole-body, complex, generating, torques, humans, humanoids \\ Disciplines \\ Engineering | Science and Technology Studies

\section{Publication Details} \\ Saab, L., Mansard, N., Soueres, P., Fourquet, J., Sreenivasa, M. \& Nakamura, Y. (2012). Whole-body torques \\ for generating complex movements in humans and humanoids. IFAC Proceedings Volumes, 45 (22), \\ 479-484. 10th IFAC Symposium on Robot Control 2012 (SYROCO 2012)

\section{Authors} \\ Layale Saab, Nicolas Mansard, Philippe Souères, J-Y Fourquet, Manish Sreenivasa, and Yoshihiko \\ Nakamura
}




\title{
Whole-body torques for generating complex movements in humans and humanoids
}

\author{
L. Saab ${ }^{*}$ N. Mansard ${ }^{*}$ P. Souères * J-Y. Fourquet ${ }^{* *}$ \\ M. Sreenivasa ${ }^{* * *}$ Y. Nakamura ${ }^{* * *}$ \\ * CNRS, LAAS, 7, avenue du Colonel Roche, F-31077 Toulouse, \\ France, Université de Toulouse(UPS) (e-mail: \\ \{lsaab,nmansard,soueres\}@laas.fr) \\ ** LGP-ENIT, 47, avenue d'Azereix, 65016, Tarbes, France, Université \\ de Toulouse (e-mail: fourquet@enit.fr) \\ *** Nakamura Laboratory, Department of Mechano-Informatics, \\ University of Tokyo, Tokyo, Japan, (e-mail: manu@ynl.t.u-tokyo.ac.jp)
}

\begin{abstract}
This paper considers the problem of identifying the control torques associated with the generation of complex movements in an anthropomorphic system. We present a generic motion generation scheme for humanoid robots. Then we use the torques estimated from human motion capture and force sensor measurements, to compare with similar movements simulated on a humanoid robot. The general features of movement during a sequence of reaching tasks are analyzed. In particular we compare kinematics-based and dynamics-based movements. Finally, the variation of torques at the different joints are compared and discussed.
\end{abstract}

Keywords: Torque control, anthropomorphic system, whole-body dynamics, quadratic programming, tasks-function, operational-space control.

\section{INTRODUCTION}

Understanding how humans plan and coordinate wholebody movements to execute complex tasks is a key question for researchers from different fields. In addition to biomechanists and neuroscientists who aim at modeling the functioning of the neuromuscular system, specialists in computer animation and roboticists try to generate human-like movements on anthropomorphic systems. We focus here on motion generation methods developed by roboticists which should not only make humanoid robots move like humans but also take into account masses and inertia in order to guarantee dynamic stability. Such algorithms are of great interest for enabling robots to execute tasks adequately in interaction with humans, but also for rehabilitation issues (Venture et al., 2007), or for ergonomic purposes (Hue et al., 2008). Sophisticated methods based on robotics tools have also been developed to reconstruct the musculoskeletal activity of the human body from the observation of its movement (Nakamura et al., 2005). These methods are based on the dynamic modeling of the human body as a polyarticulated system and reconstruction techniques using motion capture devices and additional sensor measurements.

The work presented in this paper aims at linking these two aspects of the study of anthropomorphic systems: human movement analysis and humanoid robot motion generation. We seek to develop a method for generating whole-body movements respecting dynamic stability and natural behavior by using a recently developed software at LAAS-CNRS (Saab et al., 2011b). Our study is based on a comparison of the human movements, whose parameters are reconstructed by the sDIMS simulator (Nakamura et al., 2005) developed at the YNL (University of Tokyo), with similar movements simulated by our advanced motion generation software. We seek at generating human-like motion without the need of a complex data structure. The motion generation software is based on the computation of the inverse dynamics and uses the stack of tasks formalism (Samson et al., 1991; Mansard et al., 2009). It involves a hierarchical Quadratic Programming (QP) solver (Fletcher, 1971; Escande et al., 2010), which enables the generation of whole-body movements satisfying a set of ordered constraints (Saab et al., 2011b). The reconstructed human movement and the simulated motion are compared qualitatively. We start with a simple visual comparison and then extend it to the analysis of the torques at the different joints during the motion. We also compare simulated movements obtained by considering the kinematics only, with movements generated by considering the whole dynamics.

The paper is organized as follows. The motion generation method is described in section 2 . Section 3 describes the experiments related to the human motion reconstruction and the humanoid motion generation. The results are then presented and discussed in section 4 .

\section{WHOLE-BODY MOTION GENERATION UNDER MULTI-CONSTRAINTS AND CONTACTS}

This section presents the general motion generation scheme, based on the inverse dynamics, that was used to generate whole body movements under multi-constraints and contacts. 


\subsection{The task-function approach}

The task-function approach (Samson et al., 1991), or operational-space approach (Khatib, 1987), provides a mathematical framework to describe tasks in terms of specific output functions. The usual approach consists in designing a reference behavior of the error in the task space and then applying an inverse transformation to express this reference in the configuration space. A task is defined by a triplet $\left(e, \dot{e}^{*}, Q\right)$, where $e$ is the task function that maps the configuration space to the task space, $\dot{e}^{*}$ is the reference behavior expressed in the tangent space to the task space at $e$, and $Q$ is the differential mapping between the task space and the configuration space of the robot which verifies the relation:

$$
\dot{e}+\mu=Q u,
$$

where $u$ is the control and $\mu$ is the drift of the task. To compute a specific robot control $u^{*}$ that performs the reference $\dot{e}^{*}$, any numerical inverse of $Q$ can be used ,e.g. a generalized pseudo inverse. Iteratively, a hierarchized set of tasks (or stack of tasks) can be considered, each tasks being executed within the null space of the higher-priority ones (Siciliano and Slotine, 1991; Sentis, 2007). The main drawback of this approach is the limitation to tasks defined by equalities. A solution to cope with inequalities is to use a $\mathrm{QP}$ resolution instead of a pseudo inverse to solve the linear problem.

\subsection{Generic formulation of the $Q P$ resolution scheme}

In applied mathematics, the $\mathrm{QP}$ resolution algorithm based on the active set method was first given in (Fletcher, 1971). It works as a two-stage hierarchized set, where inequalities can only be expressed at the top priority level. Slack variables are then a classical solution to circumvent this limitation (Boyd and Vandenberghe, 2004). In (Kanoun et al., 2009; Escande et al., 2010), it was proposed to extend the use of slack variables to handle more than two stages of priority. We consider that each task $\left(e_{k}, \dot{e}_{k}^{*}, Q_{k}\right)$, is associated to a pair of real numbers $\left(r_{k}^{*}, \bar{r}_{k}^{*}\right)$ defining respectively the lower and the upper bound $\mathrm{d}$ on the reference behavior. Specific cases can be easily implemented: $r^{*}=\bar{r}^{*}$ in the case of equalities, and $\underline{r}^{*}=-\infty$ or $\bar{r}^{*}=+\infty$ to handle single-bounded constraints. At stage $k$, the cascade algorithm solving the hierarchy of tasks is expressed by the following QP:

$$
\begin{array}{ll} 
& \min _{u_{k}, w_{k}}\left\|w_{k}\right\|^{2} \\
\text { s.t. } & \underline{r}_{k-1}^{*} \leq Q_{k-1} u_{k}+w_{k-1}^{*} \leq \bar{r}_{k-1}^{*} \\
& \underline{r}_{k}^{*} \leq Q_{k} u_{k}+w_{k} \leq \bar{r}_{k}^{*}
\end{array}
$$

where $Q_{k-1},\left(\underline{r}_{k-1}^{*}, \bar{r}_{k-1}^{*}\right)$ are the constraints at all the previous stages, from 1 to $k-1$, and $Q_{k},\left(r_{k}^{*}, \bar{r}_{k}^{*}\right)$ is the constraint at stage $k$. The slack variable ${ }^{1} w_{k}$ is used to add some freedom to the solver if no solution can be found when the constraint $k$ is introduced, under the $k-1$ previous constraints. Whereas $w_{k-1}^{*}$ keeps the value of the result of the optimization of the $k-1$ first QPs, $w_{k}$ is variable and can be used by the solver to relax the last

\footnotetext{
$1 w$ is an implicit optimization variable whose explicit computation can be avoided when formulating the problem as a cascade. It does not appear in the vector of optimization variables $u$. See (Escande et al., 2010) for details.
}

constraint. A solution to the strict $k-1$ constraint $Q_{k-1}$ is then always reached, even if the slack constraint $Q_{k}$ is not feasible, which corresponds to the definition of the hierarchy between tasks.

In the following, a set of tasks with a hierarchy order (or stack of tasks) will be denoted by using the lexicographic order as follows: $(i) \prec(i i) \prec(i i i) \prec \ldots$ which means that task $(i)$ has the highest priority.

\subsection{Inverse Dynamics under contact constraints}

The QP resolution scheme recalled in the preceding section is generic. It can be applied both to the kinematics (Kanoun et al., 2009) or to the dynamics (Saab et al., $2011 b$ ) of a poly-articulated system. This section and the following ones explain how this resolution scheme can be applied to the robot dynamics, including several contact forces.

The inverse dynamics model of a humanoid robot with one rigid contact with the environment can be described by a general equation of the form :

$$
A \ddot{q}+b+J_{c}^{\top} \phi_{c}=S^{\top} \tau
$$

where $A=A(q)$ and $b=b(q, \dot{q})$ are defined as the inertia matrix and the vector of nonlinear effects (Coriolis and gravity terms) respectively, $\ddot{q}$ is the vector of generalized joint accelerations ${ }^{2}, \phi_{c}=\left(f_{c}, \tau_{c}\right)$ is the generalized $6 \mathrm{D}$ contact force applied to the body in contact, expressed at the points $x_{c}, J_{c}=J_{c}(q)=\frac{\partial x_{c}}{\partial q}$ is the Jacobian matrix of the body in contact at point $x_{c}$, and $S=\left[\begin{array}{ll}0 & I\end{array}\right]$ is a matrix that allows to select the actuated joints. The rigid contact condition implies that there is no motion of the robot contact points $x_{c}$ i.e. $\dot{x}_{c}=0, \ddot{x}_{c}=0$. For a given state, it implies the linear equality constraint:

$$
J_{c} \ddot{q}=-\dot{J}_{c} \dot{q}
$$

For such a system, the differential mapping $Q$ between the task space and the configuration space is defined by the task Jacobian $J=J(q)$, computed at the configuration $q$ :

$$
J=\frac{\partial e}{\partial q}
$$

Given a task function $e$, the operational-space inversedynamic problem is to find the control input $\tau$ corresponding to an acceleration $\ddot{q}$ that will produce the reference acceleration $\ddot{e}^{*}$ in the task space. The link between the acceleration in the configuration space and the acceleration in the task space is obtained by derivating (5):

$$
\ddot{e}=J \ddot{q}+\dot{J} \dot{q}
$$

Finally, the task-function formalism requires the system to be fully constrained to ensure its stability. A very last stage is then introduced to cope with the case of an insufficient number of tasks and constraints to fulfill the full-rank condition:

$$
\ddot{q}=-K \dot{q}
$$

Using the resolution scheme described in section 2.2, the torque controller enabling the robot to follow the prescribed behaviour in the task space, while taking into

\footnotetext{
$2 \ddot{q}$ is the second order time-derivative of the vector of generalized coordinates $q=\left(q_{f}, q_{A}\right)^{T}$, where $q_{f}$ is the 6D pose of the free-floating root frame, and $q_{A}$ is the vector of joint positions
} 
account the contact force, can be determined by a cascade of QP associated with the following hierarchy of tasks: (3) $\prec(4) \prec(6) \prec(7)$. In this resolution, the variables $\phi_{c}$ and $\ddot{q}$ are then explicitly computed. The optimization variables are $u=\left(\tau, \ddot{q}, \phi_{c}\right)$.

\subsection{A sufficient condition for modeling rigid planar contacts}

To guarantee a rigid planar contact, at least three points of the body $p_{i}, i=1, \ldots, l(l \geq 3)$, not aligned, need to be considered to define the boundaries of the polygon of contact. For $i=1, \ldots, l, f_{i}$ denotes the contact force applied at the vertex $p_{i}$. Based on the demonstration in (Saab et al., 2011a), the rigid contact constraint can be expressed as follows:

$$
\begin{gathered}
Q_{c}\left[\begin{array}{l}
\phi_{c} \\
f^{\perp}
\end{array}\right]=0 \\
f^{\perp} \geq 0
\end{gathered}
$$

with $f^{\perp}=\left(f_{1}^{\perp}, f_{2}^{\perp}, \ldots, f_{l}^{\perp}\right)$ the vector of the normal components of the forces at the contact points and $Q_{c}$ a corresponding mapping matrix. The cascade of $\mathrm{QP}$ can then be re-written for the following hierarchy of tasks: (3) $\prec(4) \prec(8) \prec(6) \prec(7)$ and the optimization variables are $u=\left(\tau, \ddot{q}, \phi_{c}, f^{\perp}\right)$.

\subsection{Generalization to multiple contacts}

Eq. (3) only considers one body in planar contact. If more than one body is in contact, or if one body is in contact with more than one surface, several forces $\phi_{i}$ have to be introduced for each couple plane-body in contact:

$$
A \ddot{q}+b+\sum_{i} J_{i}^{\top} \phi_{i}=S^{\top} \tau
$$

Finally, the complete cascade for $n$ contacts and $k$ tasks is written: $(3) \prec(4.1) \prec(8.1) \prec \ldots \prec(4 . n) \prec(8 . n) \prec(6.1)$ $\prec \ldots \prec(6 . k) \prec(7)$, with the optimization variables $u=$ $\left(\tau, \ddot{q}, \phi_{1}, f_{1}^{\perp}, \ldots, \phi_{n}, f_{n}^{\perp}\right)$. This control law was integrated in the control framework SoT (Mansard et al., 2009), using the dedicated solver (Escande et al., 2010) and applied online to the humanoid robot HRP-2 (Saab et al., 2011a).

\section{HUMAN MOTION RECONSTRUCTION AND HUMANOID MOTION GENERATION}

\subsection{Human motion reconstruction}

Human movements were recorded using a Motion Analysis motion capture system consisting of 10 cameras recording a $3 \times 3 \mathrm{~m}$ free space. Participants were further instrumented with 35 reflective markers, and 16 wireless Electromyograph (EMG) electrodes (Trigno Wireless, Delsys Inc.). The recording area was fitted with two Kistler force plates $(2 \mathrm{~m} \times 0.5 \mathrm{~m})$ to measure the ground contact forces. Based on these data, the software sDIMS, developed at the YNL, which includes a complete Musculoskeletal (MS) model of the human body, was used to reconstruct the MS activity of the subject (Nakamura et al., 2005; Yamane and Nakamura, 2007). The acquisition of the markers positions was at $200 \mathrm{~Hz}$, and the data from EMG and force plates were recorded at $1 k H z$.
The experimental protocol was chosen in order to obtain interesting representative parameters of dynamic wholebody movements. The participant was asked to execute a sequence of three reaching tasks close to the limit of stability, while simultaneously satisfying additional constraints. The reaching tasks were performed with the right hand while balancing on one foot. Additionally, the left hand was holding a cup and the participant was asked to maintain its position while executing the reaching tasks. Implicitly, during each reaching task the subject was required to look at the target, although this was not stipulated in the instructions. Four snapshots of the successive phases of a trial are shown in figure 2(a). Based on the measurements given by the various sensors during this experiment, the sDIMS software was used to reconstruct the joint torques and muscle tensions. For the purpose of this study we limit ourselves to analyzing the estimated joint torques. The second phase of the study, which is described in the next section, was to reproduce the similar sequence of reaching tasks by using the motion generation software presented in section 2 .

\subsection{Humanoid motion generation}

We consider an extended dynamical model of the humanoid robot HRP-2 of LAAS-CNRS. This model contains 7 additional DOF located at the level of the torso, head, ankle and chest. The objective of our work is to show that the motion generation software presented in section 2 can be used to make this robot model achieve the same sequence of reaching tasks in a human-like way and to compare this motion to the human's. We also want to compare the movements obtained by taking into account the kinematics of the robot model only, to one with its dynamics included.

The main difficulty is to design the sequence of ordered stack of tasks to actuate and constrain the whole-body joints of the robot in order to make it execute the expected sequence of reaching tasks. In this work, the sequence of tasks was designed based on our observation of the human motion. So far, we have not developed a method for synthesizing the stack of tasks automatically. The highest priority task is to guarantee the balance of the robot. This task, which is active during the whole movement is expressed in terms of the robot center of mass (CoM). We suppose that by maintaining the projection of the CoM inside the support polygon, we can reliably reproduce the stability of the human. We choose to maintain the projection of the CoM at a fixed position with an equality constraint. Two inequality constraints are then used to limit the variation of position of the left foot and the left hand during the whole movement. This is done by maintaining the operational point of the ankle and the wrist inside a 3D polyhedron. Note however that the orientation of the left foot and the left hand are not constrained. Beside these inequality tasks, we use successive equality constraints to control the movement of the right hand toward each of the target points. However, in order to avoid auto-collisions and to increase the resemblance to human movement, we use equality tasks to drive the movement through intermediary way-points before reaching the actual targets. Equality tasks are also used to drive the gaze toward each target along the movement. The gaze task is activated 


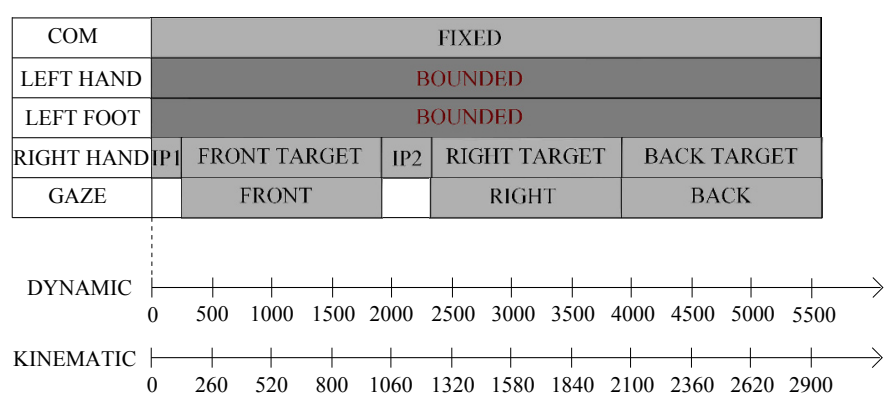

Fig. 1. Evolution of the stack of tasks designed to generate the whole-body movement of the robot.

at the end of each tracking subtask, after having reached the intermediary via-point. Actually, when observing the reference human motion, it is quite hard to determine when the subject starts looking at the target and which of the vision or the hand movement is first initiated. The choice of coupling the vision tasks with the final phase of the reaching tasks ensures the relaxation of more DOF during the pre-pointing phase.

In order to show that taking into account the dynamics is a natural way of generating movements resembling more those of humans, we applied the same sequence of stack of tasks to the kinematic and the dynamic model of the robot. Figure 1 shows the sequencing of the stack of tasks used for both simulations with the corresponding iteration numbers.In this table, the highest priority task appears at the top and the lowest priority at the bottom. IP1 and IP2 denote the two intermediary points (IP) that were added as pre-pointing tasks to avoid auto-collision between the right hand and the right foot during the first pointing task, and to avoid the collision of the right hand with the ground.

\section{RESULTS}

This section presents the human motion reconstructed with the sDIMS software, based on the MS model, and the simulated movements computed with our motion generation software, by considering successively the kinematic and the dynamic model of the robot.

\subsection{Comparison of human and robot movements}

Figure 2 shows a sequence of snapshots of the recorded human movement and the corresponding generated robot movements. The pictures on the first line show the human movement 2(a), whereas the picture on the second line 2 (b) and the third line 2(c) show the robot movement generated from the kinematic model and the dynamic model respectively. From left to right, each picture in the same column corresponds to the same phase of the movement. The first picture shows the starting position, in single support on the right foot, with a cup in the left hand. The three successive phases show the posture of the human and the robot at the end of each reaching task. Note that the gaze anticipation of the robot is not considered when computing the initial configuration. Note also that the simulation time in these resulting motions are different. In fact, the human motion generated from the recorded data has been simulated using sDIMS software while the kinematic and dynamic simulations were obtained using our developed software. The generation of the humanoid motion was based on the target definition and not on the time of reaching of each target.

During the first phase of reaching, the major differences between the robot motion generated from the kinematics and from the dynamics concern the right hand pose, the head orientation, the left foot and left hand positions, and the upper body posture. Overall, the robot motion generated from the dynamics more closely resembles the human movement than the motion generated from the kinematics. In particular, in the dynamic simulation the robot lifts his left foot in a similar manner to the human. The head movement and the hand positioning are also more similar. On the other hand, in the kinematic simulation it appears that the left foot is raised very high, the head is rotated much further, the left hand is higher and the right hand is too extended. During the reaching of the second target, we can also observe many differences. The main differences that appear in the kinematic simulation concern the position of the right knee. We observe an important flexion in the right knee which results in a lower position of the right foot. The position of the left hand, the orientation of the right hand and the head are also different. During the last phase of reaching, similar differences of flexion of the right knee occur in the kinematic simulation. However, the left arm takes different posture in the kinematic and the dynamic simulations, while respecting the bounds imposed by the inequality constraints. In the kinematic simulation, we observe that the upper body of the robot moves in the opposite direction to the target while the human body seems to move towards the target. This opposition also appears in the dynamic simulation but with less intensity.

Based on all these details, we can conclude that the movement simulated from the dynamics resembles natural human movement more than that simulated from the kinematics. This conclusion seems logical as the masses and inertia are only taken into account in the dynamics case. For a more detailed comparison of the recorded human movements and the generated robot movements, the video can be downloaded through this link:

http://homepages.laas.fr/lsaab/human-motion

\subsection{Comparison of joint torques}

In this section we compare the torques of the human movement, that were reconstructed using the sDIMS software, based on the MS model, and the torques of the robot movement that were computed based on the dynamic model of the robot. Actually, the MS model is much more detailed and accurate than the robot model, but both models have comparable masses and inertia which makes the comparison of torques possible from a qualitative point of view. As the resulting motions correspond to different simulation times, which affect the acceleration rates and therefore the torques, we do not make a quantitative analysis. In the following, each graph describing the parameters of the movements is divided into three phases corresponding to each reaching task. Let us analyze first the torques variations of the human and the robot model by considering the activity of the right leg. Comparing the human torques, shown in figure $4(\mathrm{a})$, to the robot torques of figure 


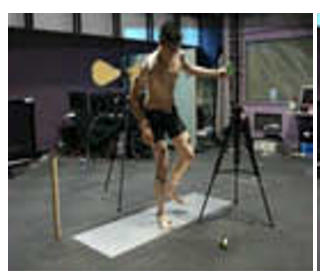

$\mathrm{t}=0 \mathrm{~s}$

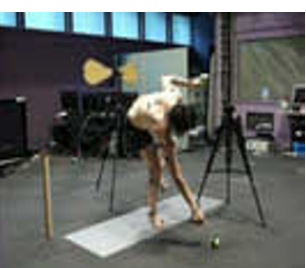

$\mathrm{t}=1.32 \mathrm{~s}$

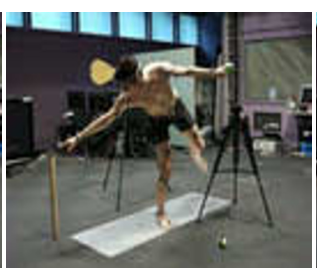

$\mathrm{t}=3.375 \mathrm{~s}$

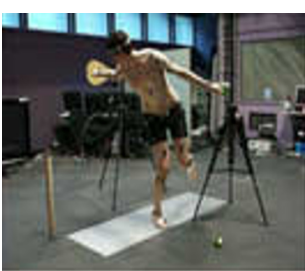

$\mathrm{t}=4.75 \mathrm{~s}$

a) Real human motion

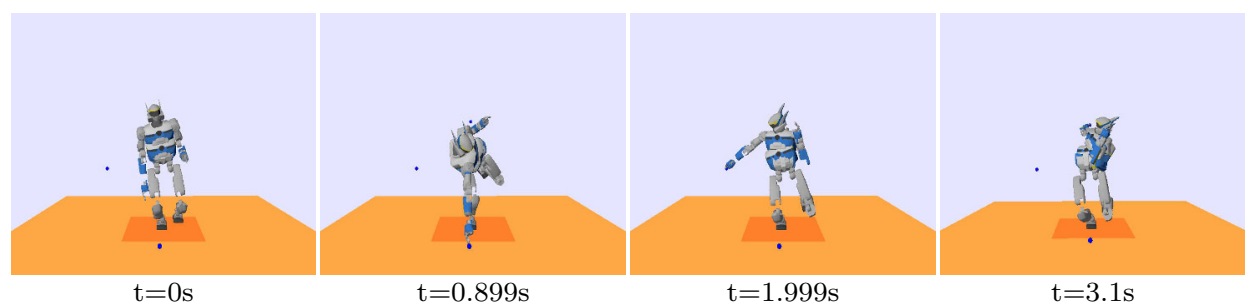

(b) Kinematic simulation

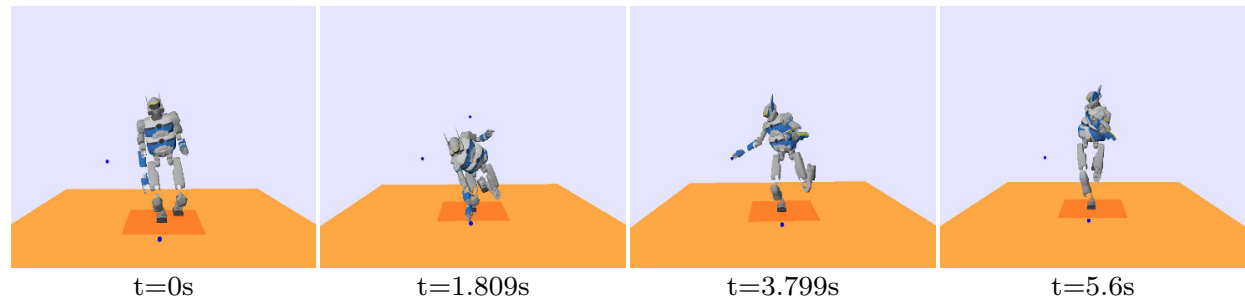

(c) Dynamic simulation

Fig. 2. Front view of the real human behavior, and the corresponding motion of the robot model in kinematic and dynamic simulations.
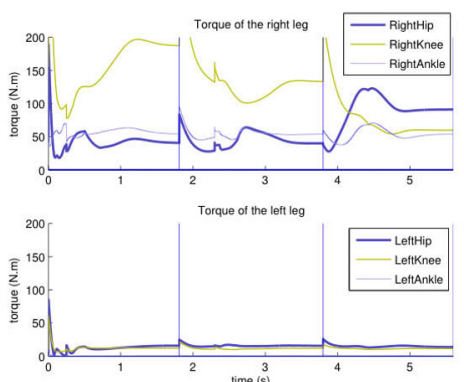

(a) Robot legs

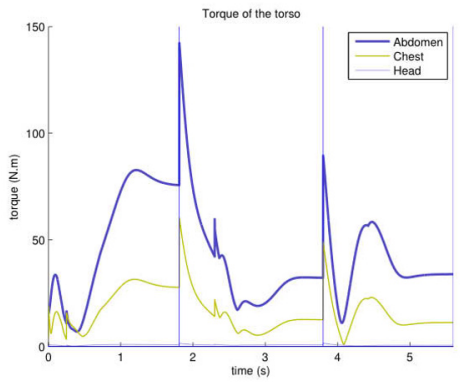

(b) Robot torso

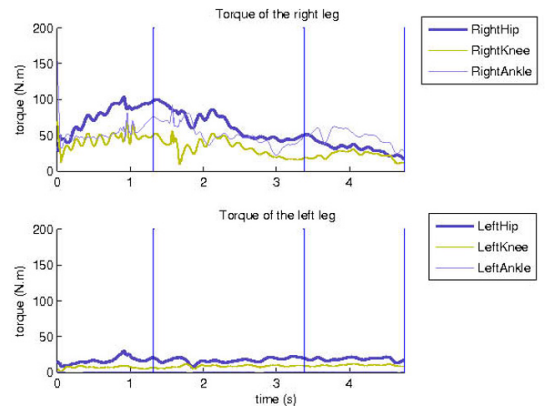

(a) Human legs

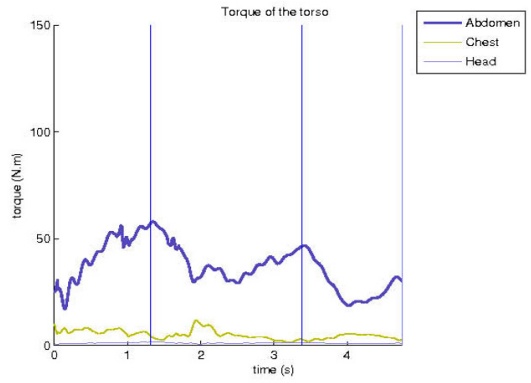

(b) Human torso
Fig. 3. The torques variation of the robot legs and torso.

3(a), we notice a similar effort decomposition and similar torque profiles. However, the maximum torque values are not associated to the same joints. So far, we have no clear explanation of this difference. Moreover, the torque profiles
Fig. 4. The torques variation of the human legs and torso computed with sDIMS.

are smooth in the case of the human, while sudden peaks appear in the case of the robot.

As shown in figures 4(a) and 3(a), the variation of torques of the left leg joints is quasi-similar in the case of the 
human and the robot model. However, here again the torques of the human motion have a smoother variation and does not present peaks as in the robot motion.

Considering the torques at the torso, it appears from figure 4(b) that the human produces high efforts at the torso joints at each transition. In the robot motion, we can see in figure $3(\mathrm{~b})$ that high peaks also occur at the transition between the three phases. Considering the chain of joints of the upper body, it appears that, from the abdomen to the head, the amplitude of torques is decreasing for both the human and the robot.

We can conclude that the most important difference between the reconstructed human movements and the generated robot movements concerns the smoothness of the torque trajectories. This interesting characteristic has to be related to the natural capacity of the humans to anticipate their movements. Whereas with the proposed motion generation method, the robot movement is guided by a succession of exponential task decays. Actually, we apply a PD control law for the convergence of the task, which induces a phase of stabilization by the end of each sequence. However, it is interesting to note that, despite the sequence of tasks, the variation of velocity is moderate. An alternative choice could be to use a task decay rate corresponding to a minimum jerk profile observed in humans (Hoff and Arbib, 1993). Inserting or removing a task, changing the priority order or considering a new target, are events that could create strong velocity variations.

\section{CONCLUSION}

This work shows that the study of anthropomorphic systems provides an interesting link between robotics and life sciences. We have presented a method that allows to generate sequences of complex human-like and dynamically stable movements. However, the motion generation method induces peaks in the torques profiles whereas the human torque trajectories are smooth. On one hand, this is due to the sequence of exponential decay of task functions and could be resolved by considering the dynamics of whole movement, instead of regulating each task individually. On the other hand, the resolution of the stack of tasks does not include any anticipation of motion, a property that naturally a human possess. Other important differences between the human movement and the simulated robot movement come from the simplicity of the considered robot model. These differences should be strongly reduced by considering a more complete and accurate anthropomorphic model.

\section{ACKNOWLEDGEMENTS}

This work is based on a collaboration between the Gepetto Group of the LAAS-CNRS and the Nakamura Laboratory $(Y N L)$ of the University of Tokyo. The authors would like to thank Ko Ayusawa and Yosuke Ikegami for assisting with the experiments and the participant for taking part in it.

\section{REFERENCES}

Boyd, S. and Vandenberghe, L. (2004). Convex Optimization. Cambridge University Press.
Escande, A., Mansard, N., and Wieber, P.B. (2010). Fast resolution of hierarchized inverse kinematics with inequality constraints. In IEEE Int. Conf. on Robotics and Automation (ICRA'10). Anchorage, USA.

Fletcher, R. (1971). A general quadratic programming algorithm. IMA Journal of Applied Mathematics, 7, 7691.

Hoff, B. and Arbib, M.A. (1993). Models of trajectory formation and temporal interaction of reach and grasp. Journal of Motor Behavior, 25(3):175-192.

Hue, V., Fourquet, J., and Chiron, P. (2008). On realistic human motion simulation for virtual manipulation tasks. In ICARCV'08, 167-172.

Kanoun, O., Lamiraux, F., Kanehiro, F., Yoshida, E., and J-P., L. (2009). Prioritizing linear equality and inequality systems: application to local motion planning for redundant robots. In IEEE Int. Conf. on Robotics and Automation (ICRA'09). Kobe, Japan.

Khatib, O. (1987). A unified approach for motion and force control of robot manipulators: The operational space formulation. International Journal of Robotics Research, $3(1), 43-53$.

Mansard, N., Stasse, O., Evrard, P., and Kheddar, A. (2009). A versatile generalized inverted kinematics implementation for collaborative working humanoid robots: The stack of tasks. In Int. Conf. on Advanced Robotics, 119. Munich, Germany.

Nakamura, Y., Yamane, K., Fujita, Y., and Suzuki, I. (2005). Somatosensory computation for man-machine interface from motion-capture data and musculoskeletal human model. IEEE Transactions on Robotics, 21(1), $58-66$.

Saab, L., Mansard, N., Keith, F., Fourquet, J., and Soueres, P. (2011a). Generation of dynamic motion for anthropomorphic systems under prioritized equality and inequality constraints. In IEEE Int. Conf. on Robotics and Automation (ICRA'11). Shangai, China.

Saab, L., Ramos, O., Mansard, N., Soueres, P., and Fourquet, J. (2011b). Generic dynamic motion generation with multiple unilateral constraints. In IEEE/RSJ Int. Conf. on Intelligent Robots and Systems (IROS'11). San Francisco, USA.

Samson, C., Le Borgne, M., and Espiau, B. (1991). Robot Control: the Task Function Approach. Clarendon Press, Oxford, United Kingdom.

Sentis, L. (2007). Synthesis and Control of Whole-Body Behaviors in Humanoid Systems. Ph.D. thesis, Stanford University, USA.

Siciliano, B. and Slotine, J.J. (1991). A general framework for managing multiple tasks in highly redundant robotic systems. In IEEE Int. Conf. on Advanced Robot. Pisa, Italy.

Venture, G., Nakamura, Y., Yamane, K., and Hirashima, M. (2007). A painless and constraint-free method to estimate viscoelastic passive dynamics of limbs' joints to support diagnosis of neuromuscular diseases. In Engineering in Medicine and Biology Society, $200 \%$. EMBS 2007. 29th Annual International Conference of the IEEE, $5362-5365$.

Yamane, K. and Nakamura, Y. (2007). Robot kinematics and dynamics for modeling the human body. International Symposium on Robotics Research. 